\title{
Solitary uterine metastasis of invasive lobular carcinoma after adjuvant endocrine therapy: a case report
}

\author{
Masafumi Toyoshima1*, Hideki Iwahashi 1,3, Takashi Shima ${ }^{1}$, Atsushi Hayasaka 1,4, Takako Kudo ${ }^{1}$, Hiromitsu Makino ${ }^{1}$, \\ Saori Igeta' ${ }^{1}$, Rui Matsuura ${ }^{1}$, Nobuko Ishigaki ${ }^{1}$, Kozo Akagi ${ }^{1}$, Junko Sakurada ${ }^{2}$, Hiroyoshi Suzuki ${ }^{2}$ \\ and Kosuke Yoshinaga'
}

\begin{abstract}
Introduction: Solitary uterine metastases from extragenital cancers are very rare. Breast cancer is the most frequent primary site of metastasis to the uterine corpus, with invasive lobular carcinoma more likely to spread to gynecologic organs than invasive ductal carcinoma.

Case presentation: A 62-year-old postmenopausal Japanese woman was diagnosed with uterine leiomyomata more than 20 years ago and had been managed conservatively until menopause. Seven years prior to her presentation, she was diagnosed with breast cancer and underwent a partial resection of her right breast for stage IIA invasive lobular carcinoma. She underwent adjuvant chemotherapy, radiotherapy, and five years of anastrozole hormonal therapy. She presented with a growing uterine mass. Her tumor marker levels were markedly increased over the course of her followup, but a systemic examination revealed only a solitary uterine tumor. She underwent a total abdominal hysterectomy with bilateral salpingo-oophorectomy. A histopathological examination, including detailed immunohistochemistry, confirmed metastatic invasive lobular carcinoma, infiltrating both her uterine myometrium and fibroid tissue.
\end{abstract}

Conclusion: We report a very rare metastatic pattern of invasive lobular carcinoma and demonstrate that gross cystic disease fluid protein-15 and mammaglobin are useful in the diagnosis of metastatic breast cancer.

Keywords: Anastrozole, Breast cancer, Immunohistochemistry, Invasive lobular carcinoma, Isolated uterine metastasis

\section{Introduction}

The ovary and the vagina are the most frequent metastatic sites for both extragenital and genital primary neoplasms, but it is very rare for extragenital cancers to metastasize to the female reproductive tract [1]. Breast cancer is the most frequent extragenital origin of metastasis to the uterine corpus [2]; however, uterine involvement remains very rare. The most common sites of breast cancer metastasis are lung, bone, liver, and brain. Most uterine metastases are found at the time of autopsy [3] because uterine involvement by metastasis is a sign of end-stage disease. When compared with invasive ductal carcinoma (IDC) of the breast, the stage-matched

\footnotetext{
* Correspondence: m-toyo@med.tohoku.ac.jp

${ }^{1}$ Department of Obstetrics and Gynecology, Sendai Medical Center, National Hospital Organization, 2-8-8, Miyagino, Miyagino-ku, Sendai, Miyagi 983-8520, Japan

Full list of author information is available at the end of the article
}

prognosis is better for patients with invasive lobular carcinoma (ILC) [4]. However, ILC is more likely to spread to gynecologic organs $[5,6]$.

Anastrozole, a third-generation aromatase inhibitor, has been shown to be superior to tamoxifen as adjuvant therapy for patients with breast cancer [7]. In addition, anastrozole is expected to be safer for the prevention of recurrence and metastasis, because tamoxifen increases the risk of endometrial cancer [8].

We report the case of a patient with enlarging uterine fibroids after menopause. Seven years prior, she had been treated for ILC with surgery, radiotherapy, and anastrozole hormonal therapy. She underwent a total hysterectomy, and a pathological examination at this time revealed a solitary metastatic ILC lesion involving the uterine myometrium and infiltrating a fibroid. 


\section{Case presentation}

A 62-year-old postmenopausal Japanese woman presented with a $23 \times 12 \mathrm{~cm}$ uterine mass. The mass was growing, and our patient reported abdominal compression. She had first been diagnosed with uterine leiomyomata more than 20 years previously and had taken gonadotropin-releasing hormone agonist therapy for six months. Her age at menopause was 51 years and at the age of 55 years, she was diagnosed with breast cancer and underwent a partial resection of her right breast. A pathological examination of the neoplasm revealed ILC, classified as pT2N1M0, stage IIA. Immunohistochemical staining was positive for estrogen receptors, progesterone receptors, and cerbB2, and $20 \%$ of the cells were positive for Ki-67. She received adjuvant radiotherapy, 50 Gy to her right breast and an additional 10 Gy for localized irradiation. Our patient also underwent adjuvant chemotherapy with six cycles of fluorouracil, epirubicin, and cyclophosphamide. In addition, she took anastrozole as hormonal therapy for five years after surgery.

Twenty months after the therapy ended, she was noted to have elevated tumor markers (cancer antigen 15-3, 87.9IU/mL; carcinoembryonic antigen (CEA), $15.6 \mathrm{ng} / \mathrm{mL}$; National Cancer Center stomach 439, 120IU/mL). A diagnostic workup was undertaken that included computed tomography, magnetic resonance imaging (Figure 1a), and transvaginal ultrasonography. Her uterus was irregularly enlarged, measuring $23 \times 12 \mathrm{~cm}$. Despite her postmenopausal status, the uterine leiomyomata had markedly enlarged since the previous examination two years prior. A systemic examination, including technetium 99m hydroxymethylene diphosphonate bone scintigraphy, positron emission tomography-computed tomography (Figure 1b), and upper endoscopy, revealed no other lesions.
Endometrial sampling was technically impossible given the size of the mass.

Our patient underwent a total abdominal hysterectomy and bilateral salpingo-oophorectomy with the diagnosis of uterine malignancy, although it was unknown whether the tumor was primary or metastatic. A pathological examination revealed malignant cells with poor epithelial staining, along with irregular nuclei; these cells were infiltrating both her uterine fibroid tissue and her myometrium (Figure 2a). There was a small nest of malignant cells in the stroma of the atrophic endometrium. Immunohistochemical staining was positive for estrogen receptors, cytokeratin AE1/AE3, anti-cytokeratin CAM5.2, cluster of differentiation (CD) 10, CEA, cytokeratin (CK) 7, gross cystic disease fluid protein-15 (GCDFP-15), and mammaglobin (Figure 2b). The tumor was negative for progesterone receptors, CD45, myeloperoxidase, CD68, chromogranin A, synaptophysin, CD56, vimentin, desmin, smooth muscle actin, CK20, CDX-2, and E-cadherin. The previous breast tissue specimens were also examined, and malignant cells with a similar form to those seen in the uterine tumor were observed (Figure 2c). The histopathological diagnosis was metastasis of breast ILC to both the uterine leiomyoma and the myometrium. Our patient is currently undergoing exemestane hormonal therapy and remains without evidence of recurrent disease.

\section{Discussion}

ILC accounts for about $10 \%$ of breast cancer in female patients [9]. The sites of metastatic spread in ILC differ from those of IDC: in the latter, the common metastatic sites are the lung, bones, liver, and brain; in ILC, the gastrointestinal tract, peritoneum, retroperitoneum, and gynecologic organs have been reported as metastatic

\section{(a)}

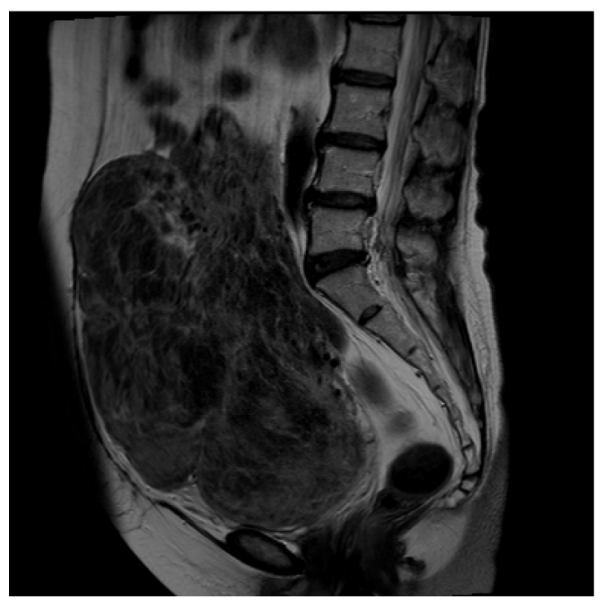

(b)

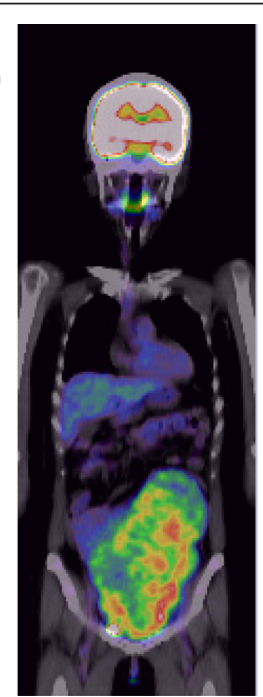

Figure 1 Radiological images. (a) Pelvic magnetic resonance imaging, T2-enhanced sagittal section, showing an irregularly enlarged uterine tumor. (b) Positron emission tomography-computed tomography showing strong uptake associated with the uterine tumor. 
(a)

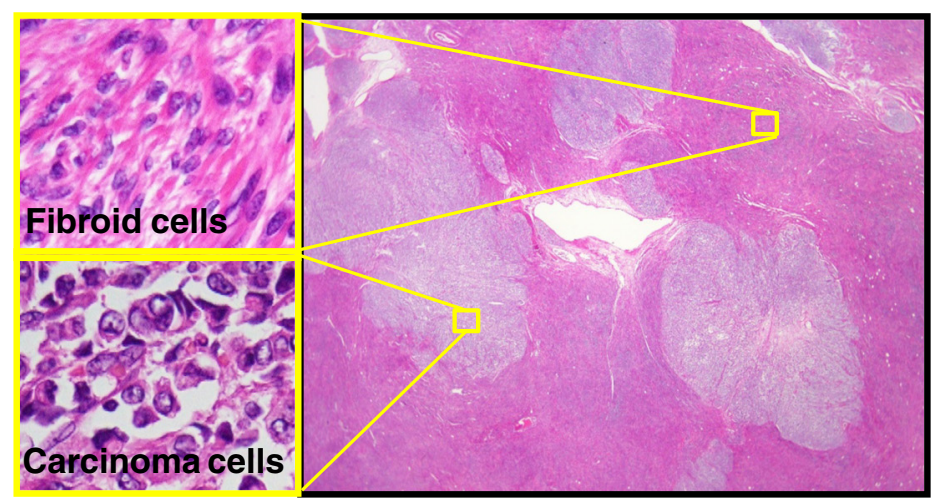

(b)

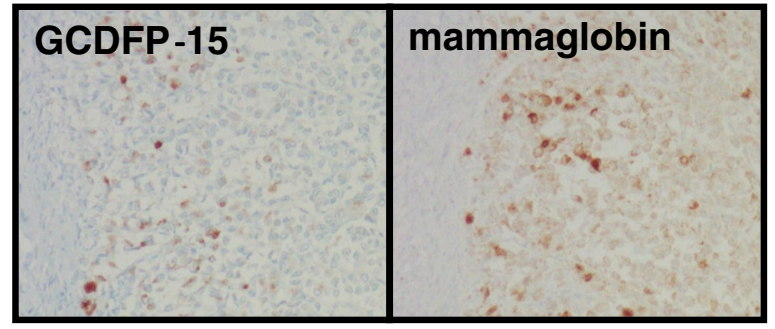

(c)

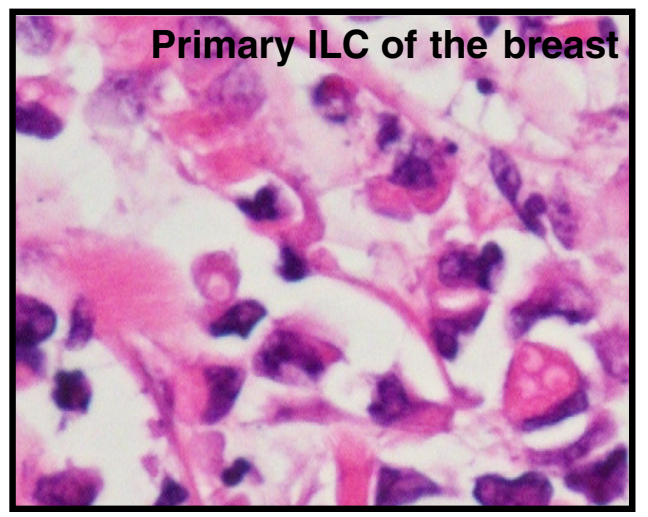

Figure 2 Pathological microscopic examination. (a) Clusters of malignant epithelial cells infiltrate the uterine fibroma. Staining: hematoxylin and eosin. Top left: magnified image of fibroid cells; bottom left: magnified image of carcinoma cells. (b) Immunohistochemical staining positive for gross cystic disease fluid protein-15 (GCDFP-15) and mammaglobin. (c) Hematoxylin and eosin staining of the primary breast cancer specimen demonstrates poor epithelial staining of the malignant cells with irregular nuclei. ILC, invasive lobular carcinoma.

sites [5]. Anastrozole is considered to be better for adjuvant therapy than tamoxifen, especially for postmenopausal patients with hormone-sensitive early breast cancer [7]. The anticancer effects of anastrozole have also been studied in endometrial cancers in a randomized pilot study [10]. Despite the fact that there is a wide range of metastatic sites possible in patients with ILC, it is extremely rare to see solitary uterine involvement after anastrozole therapy for the disease; just a few case reports have been published in the English literature [11-13]. These reports emphasize the usefulness of GCDFP-15, known for having high sensitivity and specificity for breast cancer, as a tumor marker at the metastatic site [11-13]. In our patient, the malignant epithelial cells within her uterus showed poor epithelial staining and had little cytoplasm and irregular nuclei. The pathological diagnosis was very difficult on the basis of hematoxylin and eosin staining alone: a wide differential diagnosis was possible, including a blood-derived tumor, a neuroendocrine tumor, a sarcoma, and small cell carcinoma. However, immunohistochemistry positivity for cytokeratin, estrogen receptors, progesterone receptors, GCDFP-15, and mammaglobin gave definitive information by which we could make the diagnosis of metastatic ILC to the uterus.

If metastatic infiltration involves the endometrial glands, then abnormal uterine bleeding is often the first symptom, and endometrial sampling allows the detection of malignant cells [14]. However, patients may often 
be asymptomatic when the infiltration affects only the myometrium, as in our patient. When tumors metastasize to the uterus, they involve only the myometrium in $64.5 \%$ of cases, both the myometrium and the endometrium in $32.7 \%$, and only the endometrium in just 3.8\% [1]. Our patient presented with abdominal distension but without abnormal bleeding. Furthermore, a huge uterine fibroid prohibited endometrial sampling. Hence, clinicians should keep in mind that metastasis of malignant cells to the uterus does not always result in 'typical symptoms' such as abnormal genital bleeding.

\section{Conclusions}

We report a very rare metastatic pattern of ILC and demonstrate that detailed immunohistochemistry, including GCDFP-15 and mammaglobin, is useful in the diagnosis of metastatic breast cancer. It is important for clinicians to recognize that ILC and IDC have different patterns of metastasis and that an isolated uterine metastasis may occur after either anastrozole or tamoxifen therapy. A comprehensive gynecologic workup should be considered in patients with breast cancer who have uterine lesions.

\section{Consent}

Written informed consent was obtained from the patient for publication of this case report and accompanying images. A copy of the written consent is available for review by the Editor-in-Chief of this journal.

\section{Abbreviations}

CD: cluster of differentiation; CEA: carcinoembryonic antigen; CK: cytokeratin; GCDFP-15: gross cystic disease fluid protein-15; IDC: invasive ductal carcinoma; ILC: invasive lobular carcinoma.

\section{Competing interests}

The authors declare that they have no competing interests.

\section{Authors' contributions}

MT analyzed and interpreted the patient data, drafted the manuscript, and created the figures. JS and HS performed the histological examination, made the pathological diagnosis, and revised the manuscript. HI, TS, AH, TK, HM, SI, $\mathrm{RM}, \mathrm{NI}$, and KA performed physical examinations and provided medical care to the patient. TK and KA provided valuable insight during manuscript preparation. KY supervised the entire case. All authors read and approved the final manuscript.

\section{Acknowledgements}

The authors thank Dr Takashi Toshima and Dr Mii Shibahara for providing medical information, and Dr Takanori Watanabe and Dr Maiko Okano for their critical review of the manuscript.

\section{Author details}

'Department of Obstetrics and Gynecology, Sendai Medical Center, National Hospital Organization, 2-8-8, Miyagino, Miyagino-ku, Sendai, Miyagi 983-8520, Japan. ${ }^{2}$ Department of Pathology, Sendai Medical Center, National Hospital Organization, Sendai, Japan. ${ }^{3}$ Department of Obstetrics and Gynecology, Self-Defense Force Sendai Hospital, Sendai, Japan. ${ }^{4}$ Department of Obstetrics and Gynecology, Sendai City Hospital, Sendai, Japan.

Received: 25 November 2014 Accepted: 30 December 2014

Published online: 14 February 2015

\section{References}

1. Mazur MT, Hsueh S, Gersell DJ. Metastases to the female genital tract. Analysis of 325 cases. Cancer. 1984;53:1978-84.

2. Kumar NB, Hart WR. Metastases to the uterine corpus from extragenital cancers. A clinicopathologic study of 63 cases. Cancer. 1982;50:2163-9.

3. Di Bonito L, Patriarca S, Alberico S. Breast carcinoma metastasizing to the uterus. Eur J Gynaecol Oncol. 1985;6:211-7.

4. Wasif N, Maggard MA, Ko CY, Giuliano AE. Invasive lobular vs. ductal breast cancer: a stage-matched comparison of outcomes. Ann Surg Oncol. 2010;17:1862-9.

5. Harris M, Howell A, Chrissohou M, Swindell Rl, Hudson M, Sellwood RA. A comparison of the metastatic pattern of infiltrating lobular carcinoma and infiltrating duct carcinoma of the breast. Br J Cancer. 1984;50:23-30.

6. Lamovec J, Bracko M. Metastatic pattern of infiltrating lobular carcinoma of the breast: an autopsy study. J Surg Oncol. 1991;48:28-33.

7. Baum M, Budzar AU, Cuzick J, Forbes J, Houghton JH, Klijn JGM, et al. Anastrozole alone or in combination with tamoxifen versus tamoxifen alone for adjuvant treatment of postmenopausal women with early breast cancer: first results of the ATAC randomised trial. Lancet. 2002;359:2131-9.

8. Ismail SM. Gynaecological effects of tamoxifen. J Clin Pathol. 1999;52:83-8.

9. Fisher ER, Gregorio RM, Fisher B, Redmond C, Vellios F, Sommers SC. The pathology of invasive breast cancer. A syllabus derived from findings of the National Surgical Adjuvant Breast Project (protocol no. 4). Cancer. 1975;36:1-85.

10. Thangavelu A, Hewitt MJ, Quinton ND, Duffy SR. Neoadjuvant treatment of endometrial cancer using anastrozole: a randomised pilot study. Gynecol Oncol. 2013;131:613-8.

11. Erkanli S, Kayaselcuk F, Kuscu E, Bolat F, Sakalli H, Haberal A. Lobular carcinoma of the breast metastatic to the uterus in a patient under adjuvant anastrozole therapy. Breast. 2006;15:558-61.

12. Ustaalioglu BBO, Bilici A, Seker M, Salman T, Gumus M, Barisik NO, et al. Metastasis of lobular breast carcinoma to the uterus in a patient under anastrozole therapy. Onkologie. 2009;32:424-6.

13. Arslan D, Tural D, Tatlı AM, Akar E, Uysal M, Erdoğan G. Isolated uterine metastasis of invasive ductal carcinoma. Case Rep Oncol Med. 2013;2013:793418.

14. Piura B, Yanai-Inbar I, Rabinovich A, Zalmanov S, Goldstein J. Abnormal uterine bleeding as a presenting sign of metastases to the uterine corpus, cervix and vagina in a breast cancer patient on tamoxifen therapy. Eur J Obstet Gynecol Reprod Biol. 1999;83:57-61.

\section{Submit your next manuscript to BioMed Central and take full advantage of:}

- Convenient online submission

- Thorough peer review

- No space constraints or color figure charges

- Immediate publication on acceptance

- Inclusion in PubMed, CAS, Scopus and Google Scholar

- Research which is freely available for redistribution 